\title{
Autonomic dysreflexia during bowel evacuation procedures and bladder filling in subjects with spinal cord injury
}

\author{
PM Faaborg ${ }^{1,2}$, P Christensen ${ }^{1}$, A Krassioukov ${ }^{3}$, S Laurberg $^{1}$, E Frandsen ${ }^{4}$ and K Krogh ${ }^{2}$
}

\author{
Study design: Randomized, controlled clinical trial. \\ Objectives: Bladder and bowel management may cause serious autonomic dysreflexia (AD) in subjects with high spinal cord injury \\ (SCI). We aimed at investigating autonomic responses to digital rectal evacuation (DE), transanal irrigation (TAI) with $500 \mathrm{ml}$ and \\ filling cystometry (FC) in $\mathrm{SCl}$.
}

Setting: Aarhus University Hospital, Denmark.

Methods: Eight subjects with SCI (AIS A) at or above T6 (high SCI) and a previous history of AD were compared with three subjects with $\mathrm{SCl}$ (AIS A) between T10 and L2 (low SCI). In randomized order, DE, TAI and FC were performed. AD was defined as an acute rise in systolic blood pressure (SBP) of $\geqslant 30 \mathrm{~mm} \mathrm{Hg}$ above baseline. Blood levels of norepinephrine and epinephrine were determined before and shortly after the procedures.

Results: During all three procedures, $\mathrm{AD}$ occurred in all patients with high $\mathrm{SCl}$ but not in those with low $\mathrm{SCl}$. In high $\mathrm{SCl}$ subjects, DE increased median sBP from 127 (range: 86-154) to 188 (range: 140-206) mm Hg ( $P<0.02)$, TAl from 126 (range: 91-146) to 163 (range: $130-188) \mathrm{mm} \mathrm{Hg}(P<0.02)$ and FC from 125 (range: 106-149) to 200 (range: 179-220) $\mathrm{mm} \mathrm{Hg}(P<0.01)$. The sBP increase was lower during TAI than during DE $(P<0.05)$ or FC $(P<0.02)$. In high SCI subjects, the blood levels of norepinephrine, but not those of epinephrine, increased significantly during all three stimuli (all $P<0.05$ ).

Conclusion: Bowel and bladder management caused AD in high SCI. The response is less severe during TAI than during FC or DE. Spinal Cord (2014) 52, 494-498; doi:10.1038/sc.2014.45; published online 29 April 2014

\section{INTRODUCTION}

Autonomic dysreflexia $(\mathrm{AD})$ is a common and potentially lifethreatening complication to spinal cord injury (SCI) at or above the sixth spinal cord thoracic segment (T6). ${ }^{1}$ It is a constellation of signs and symptoms including headache, feeling of anxiety, bradycardia, cardiac arrhythmias, profuse sweating above the level of injury, piloerection and flushing, pale and dry skin below the level of injury, blurred vision and nasal congestion ${ }^{2}$ occurring in response to usually noxious, but sometimes also nonnoxious, stimuli below the level of spinal cord lesion. The pathophysiology of $\mathrm{AD}$ is not completely understood but includes widespread uncontrolled sympathetic activation followed by vasoconstriction and increased blood pressure $(\mathrm{BP}) .^{3}$ The clinical presentation of $\mathrm{AD}$ varies from asymptomatic $^{4}$ to life-threatening emergencies with systolic blood pressure $(\mathrm{sBP})$ of $\geqslant 300 \mathrm{~mm} \mathrm{Hg}^{2}$

Neurogenic bowel and bladder are common consequences of SCI. Both rank among the top three symptoms affecting the quality of life of patients with SCI. ${ }^{5}$ Clean intermittent catheterization is arguably the preferred approach for neurogenic bladder management in people with SCI at or above T12. ${ }^{6}$ Neurogenic bowel dysfunction may prove more difficult in clinical practice and most subjects with SCI need digital anorectal stimulation/evacuation or suppositories to induce defecation. ${ }^{5,7}$ Transanal irrigation (TAI) with enema administered through special rectal catheters results in evacuation of the distal colorectum $^{8,9}$ and has proven effective for severe neurogenic bowel dysfunction. ${ }^{8,10}$ TAI is commonly used in Europe and it has recently been introduced in the United States for management of neurogenic bowel dysfunction. Usually, $500-1000 \mathrm{ml}$ of lukewarm tap water is installed every to every third day and for most patients the procedure takes between 30 and $45 \mathrm{~min}$.

Otherwise unrecognized stimuli from the bladder and gut are common causes of $\mathrm{AD}^{11}$ and many patients have symptoms of $\mathrm{AD}$ during standard bowel emptying procedures. ${ }^{5,8}$ In spite of this, autonomic responses to bladder filling and bowel emptying procedures have only been scarcely investigated. In addition, it is unknown whether irrigation, usually with $\sim 500-1000 \mathrm{ml}$, carries a higher risk of inducing $\mathrm{AD}$ than standard bowel emptying procedures. Accordingly, the aim of the present study was to describe and compare autonomic responses during (1) bladder filling, (2) digital anorectal stimulation/evacuation and (3) TAI in patients with SCI.

\section{MATERIALS AND METHODS}

Subjects

Adult SCI patients were consecutively evaluated for inclusion from the Anorectal Physiology Unit, Aarhus University Hospital or from the Spinal Cord Injury Clinic, Viborg Hospital, Denmark. Patients were considered for inclusion if they either had (1) complete SCI (AIS A) at or above

${ }^{1}$ Pelvic Floor Unit, Department of Surgery P, Aarhus University Hospital, Aarhus, Denmark; ${ }^{2}$ Neurogastroenterology Unit, Department of Hepatology and Gastroenterology, Aarhus University Hospital, Aarhus, Denmark; ${ }^{3}$ Division of Physical Medicine and Rehabilitation, ICORD, Department of Medicine, University of British Columbia, Vancouver, British Columbia, Canada and ${ }^{4}$ Department of Clinical Physiology and Nuclear Medicine, Glostrup University Hospital, Copenhagen, Denmark

Correspondence: Dr PM Faaborg, Pelvic Floor Unit, Department of Surgery P, Aarhus University Hospital, Tage-Hansensgade 2, Aarhus C DK-8000, Denmark. E-mail: pmfaaborg@gmail.com

Received 10 October 2013; revised 27 January 2014; accepted 3 February 2014; published online 29 April 2014 
the sixth spinal cord thoracic level (T6) and a previous history of AD or (2) complete SCI (AIS A) between tenth thoracic (T10) and second lumbar (L2) segments. Exclusion criteria were previous major intraabdominal surgery, systemic medication altering bowel or bladder function, major psychiatric disease, inability to understand information and give informed consent and current disease impairing participation (for example, pressure ulcer, severe spasticity or urinary tract infection). Thorough instruction with emphasis on safety and treatment of $\mathrm{AD}$ was given to all participants and staff. The study was performed in accordance with the Helsinki II declaration and was approved by the Ethics Committee, Central Denmark Region (ID 2009-0112). The study was registered on ClinicalTrials.gov (ID NCT01059370).

Among 57 patients referred to the two centers, 34 fulfilled the inclusion criteria. Nine with high SCI and three with low SCI accepted to participate. Baseline information obtained included The International Bowel Function Basic Spinal Cord Injury Data Set, ${ }^{12}$ The Autonomic Standard Assessment Form ${ }^{13}$ and demographic data including age, gender and use of medication. Three patients with high SCI were treated with baclofen administrated with an intrathecal pump. A 4-week diary of daily AD episodes was completed before and after trial participation.

\section{Experimental protocol}

All examinations were performed in the fasting state and in a standard setting with at least $4 \mathrm{~h}$ between examinations. The order of examinations was random and determined by drawing a sealed envelope allocating 2 of the 12 subjects to each of the six possible orders of three stimuli.

For bowel emptying procedures participants were seated on a commode in the upright position. For the filling cystometry they were seated as upright as possible in a hospital bed. Throughout the three examinations blood pressures and heart rate were monitored with the Finometer PRO (Finapres Medical Systems BV, Amsterdam, The Netherlands). The Finometer PRO determines blood pressure for every contraction of the heart. The filling cystometry (FC) was performed with Menuet (Dantec, Skovlunde, Denmark). Three blood samples were taken for analysis of norepinephrine and epinephrine levels: (1) after $10 \mathrm{~min}$ of stable baseline BP and heart rate measurements, (2) $3 \mathrm{~min}$ after maximum stimulation and (3) 12 min after maximum stimulation. As a precaution, poststimulation $\mathrm{BP}$ and heart rate were monitored until reaching baseline levels.

Digital anorectal stimulation/evacuation and TAI were performed by the same experienced bowel specialist nurse. All participants had an empty bladder during bowel evacuations. The FC was performed by another bowel and bladder specialist nurse. Examinations were performed in a standard manner as recommended by Coggrave and Norton. ${ }^{14}$ Thus, digital rectal evacuation (DE) was performed once or twice lasting no longer than 5 to $10 \mathrm{~min}$. TAI was performed with the Peristeen anal irrigation system (Coloplast AS, Humlebaek, Denmark). The bowel nurse inserted the catheter into the rectum of the patient, inflated the catheter balloon with 30 to $60 \mathrm{ml}$ of air, and installed $500 \mathrm{ml}$ lukewarm water into the rectum. Administration of the enema lasted 5-10 min. Afterwards the catheter was removed and the enema and stools would empty into the commode. FC was performed on an empty bladder with infusion of tepid, sterile saline at a rate of $50 \mathrm{ml}$ per min. The procedure was stopped when a maximum of $500 \mathrm{ml}$ was installed, or when reaching the individual previously determined FC value. All three examinations were terminated after standard stimulation if severe subjective symptoms of autonomic dysreflexia occurred, or if sBP increased by $75 \%$ of resting values. Maximum stimulation was defined for each of the three stimuli as the time when (1) $500 \mathrm{ml}$ had been installed in the bowel (transanal irrigation), (2) the FC volume or the maximum of $500 \mathrm{ml}$ had been reached (bladder filling) and (3) just before digital stimulation was stopped (digital stimulation). Autonomic dysreflexia was defined as an increase in sBP of at least $30 \mathrm{~mm} \mathrm{Hg}$ above baseline. ${ }^{1}$

Epinephrine and norepinephrine were measured by radioimmunoassay using a commercial kit (2-Cat RIA, Labor Diagnostika Nord, Nordhorn, Germany). Intra- and inter-assay variations for the analyses are $4.6 \%$ and $6.1 \%$ respectively.

\section{Statistical analysis}

Data analysis was performed with the computer program STATA/IC 10 for Windows (StataCorp, College Station, TX, USA). As data deviated from the normal distribution (determined by normal probability plots), the nonparametric Wilcoxon's signed-rank test was used for comparisons. Descriptive statistics were performed on background data. $P<0.05$ was considered statistically significant.

\section{RESULTS}

Eight subjects with SCI (AIS A) at or above T6 and three with SCI (AIS A) at T10-L2 completed the study according to protocol after reaching maximum stimulation. For this paper, the two groups will be referred to as high SCI and low SCI, respectively. One participant with high SCI did not complete the study as she had severe AD early in her first examination. For safety reasons she was excluded from further study. At baseline with subjects sitting in the upright position just before the first examination, one participant with high SCI had mild hypertension $(154 / 88 \mathrm{~mm} \mathrm{Hg})$ and one with low SCI had moderate/ severe hypertension $(181 / 99 \mathrm{~mm} \mathrm{Hg})$. All signs of AD had disappeared before the subjects left the unit. Demographics are presented in Table 1 and baseline autonomic and gastrointestinal functions in Tables 2 and 3.

All eight remaining participants with high SCI had AD during all examinations (Figure 1 and Table 4). However, sBP increased less during TAI $(36 \mathrm{~mm} \mathrm{Hg}$, range 30-63) than during digital evacuation (57 mm Hg, range $41-75 ; P<0.05)$ or $\mathrm{FC}(61 \mathrm{~mm} \mathrm{Hg}$, range 55-100; $P<0.02)$. The difference in sBP response between digital evacuation and FC was nonsignificant $(P=0.09)$. Median 'time at risk', defined as sBP above $150 \%$ of baseline sBP, was 3 min (range 2-14) during digital evacuation, $4 \mathrm{~min}$ (range 2-9) during transanal irrigation and 4 min (range $2-7$ ) during filling cystometry $(P>0.53)$. During all three procedures, subjective symptoms of $\mathrm{AD}$ occurred in seven of eight subjects with high SCI. One participant with a traumatic T5 (AIS A) lesion had asymptomatic AD during all three procedures.

According to our definition of $\mathrm{AD}$ as an increase of at least $30 \mathrm{mmHg}$ above sBP, none of the subjects with low SCI had AD or symptoms of $\mathrm{AD}$ during any of the procedures. There was no difference in sBP response during TAI $(16 \mathrm{~mm} \mathrm{Hg}$, range 12-18)

\section{Table 1 Demographic data}

\begin{tabular}{|c|c|c|}
\hline & $\begin{array}{l}\text { Subjects with high SCl } \\
\quad(\mathrm{n}=8,6 \mathrm{M} \text { and } 2 \mathrm{~F})\end{array}$ & $\begin{array}{l}\text { Subjects with low SCl } \\
(\mathrm{n}=3,2 \mathrm{M} \text { and } 1 \mathrm{~F})\end{array}$ \\
\hline Age in years (median, range) & $52(41-69)$ & $51(47-57)$ \\
\hline Years since $\mathrm{SCl}$ (median, range) & $17(3-27)$ & $9(7-26)$ \\
\hline \multirow[t]{6}{*}{ Level of complete injury (AIS A) } & C5 in 1 & T10 in 1 \\
\hline & C6 in 2 & $\mathrm{~L} 2$ in 2 \\
\hline & T3 in 1 & \\
\hline & T4 in 1 & \\
\hline & T5 in 2 & \\
\hline & T6 in 1 & \\
\hline \multicolumn{3}{|l|}{ Traumatic etiology } \\
\hline Traffic accident & 6 & 1 \\
\hline Fall & 2 & \\
\hline \multicolumn{3}{|l|}{ Nontraumatic } \\
\hline Disc disease & & 1 \\
\hline Tumor & & 1 \\
\hline AD occurring daily $(n, \%)$ & $3(37.5 \%)$ & None \\
\hline
\end{tabular}

Abbreviations: $A D$, autonomic dysreflexia; F, female; $\mathrm{M}$, male; $\mathrm{SCl}$, spinal cord injury. 
Table 2 Autonomic function

\begin{tabular}{lrr}
\hline The Autonomic Standard & Subjects with & Subjects with \\
Assessment Form & high SCl $(\mathrm{n}=8)$ & low SCl $(\mathrm{n}=3)$
\end{tabular}

$H R$

Normally controlled

Tachycardia

$B P$

Normally controlled

AD

0

8

Sweating

Normally controlled

Hyperhydrosis above lesion

Hyperhydrosis below lesion

Hypohydrosis below lesion

Temperature

Normally controlled

Hyperthermia

Hypothermia

Unknown

Bronchopulmonary system

Normally controlled

Full ventilatory support

Impaired, no vent support

Bladder

Awareness to empty bladder

Ability to prevent leakage

Intermittent catherization

Suprapubic catheter

Urinary sheath

Bowel

Sensation of bowel movement

Continence

Voluntary sphincter contraction

Sexual function

Genital arousal

\begin{tabular}{lcc} 
Orgasm & 2 (2 reduced $)$ & 1 \\
Ejaculation & 2 (2 reduced $)$ & 0 \\
Sensation of menses & 1 (1 indirect) & 1 (1 indirect) \\
\hline
\end{tabular}

Abbreviations: $\mathrm{AD}$, autonomic dysreflexia; $\mathrm{BP}$, blood pressure; $\mathrm{HR}$, heart rate; $\mathrm{SCl}$, spinal cord injury.

Autonomic function among patients included in the study assessed with the Autonomic

Standards Assessment Form (22) is shown.

compared with digital evacuation $(15 \mathrm{~mm} \mathrm{Hg}$, range $13-22 ; P=0.28)$ or $\mathrm{FC}(12 \mathrm{~mm} \mathrm{Hg}$, range $11-17 ; P=0.10)$.

After all three procedures, blood levels of norepinephrine taken 3 min after maximum stimulation were significantly above baseline in subjects with high SCI (Table 4). There was no difference between the levels reached during the three stimuli $(P>0.60)$. At $12 \mathrm{~min}$ after maximum stimulation, levels of norepinephrine were no longer statistically higher than at baseline (Table 4). Blood levels of
Table 3 Bowel function

\begin{tabular}{lrr}
\hline The International Bowel Function & 8 Participants & 3 Participants \\
Basic Spinal Cord Injury Data Set & with high SCl (n) & with low SCl (n) \\
\hline
\end{tabular}

GI or anal dysfunction before $\mathrm{SCl}$

Abdominal surgical procedures

0

Awareness of the need to defecate

Indirect

$\begin{array}{ll}0 & 0 \\ 0 & 0 \\ 4 & 3\end{array}$

Defecation method (main)

Straining/bearing down

Transanal irrigation

Mini enema ( $\leqslant 150 \mathrm{ml}$ )

Suppositories

Digital stimulation or evacuation

3

Defecation method (supplementary)

Digital stimulation or evacuation

Suppositories

$7 \quad 3$

Time required for defecation

0-5 min

11-20 min

21-30 min

31-60 min

$>60 \mathrm{~min}$

Frequency of defecation

Once a day

Not daily but more than twice a week

Not every week but more than once a month

$0 \quad 1$

30

3

0

2

0

1

Frequency of fecal incontinence

Daily

Not daily but at least once a week

Not every week but more than once a month

Less than once a month

Never

Need to wear pad or plug

Daily

Not every week but at least once a month

Medication affecting bowel function

Oral laxative use

Osmotic laxatives

Irritant laxatives

Perianal problems

Hemorrhoids

Perianal sores

Fissures

$\begin{array}{ll}1 & 0 \\ 2 & 2 \\ 2 & 1 \\ 1 & 0 \\ 2 & 0\end{array}$

Abbreviations: $\mathrm{Gl}$, gastrointestinal; $\mathrm{SCl}$, spinal cord injury.

Bowel function among patients included in the study assessed with the International Bowel Function Basic Spinal Cord Injury Data Set (21) is shown.

epinephrine did not increase significantly above baseline values during any of the procedures $(P>0.16)$.

In patients with low SCI, blood levels of norepinephrine and epinephrine did not increase during investigations.

In comparing data from dairies in the weeks before and after investigations, no consistent change in $\mathrm{AD}$ symptoms was found. 


\section{DISCUSSION}

In the present study, all subjects with high SCI had AD during bladder filling, digital anorectal stimulation and transanal colorectal irrigation. Our study for the first time demonstrated a significant difference in the severity of $\mathrm{AD}$ in the same population with various bladder- and bowel-related procedures. Stimuli from the lower urinary tract are generally considered the most common causes of $\mathrm{AD}$. During standard urodynamic examinations, it has been reported that $43 \%$ of patients with lesions at or above T6 and 15\% of patients with lesions below have AD. ${ }^{15}$ In a retrospective study of 571 Japanese patients with high SCI, symptomatic AD was mainly associated with use of reflex voiding and manual removal of stool. ${ }^{16}$ In contrast, subjects who were continent on spontaneous voiding and defecation had the lowest prevalence of $\mathrm{AD}$.

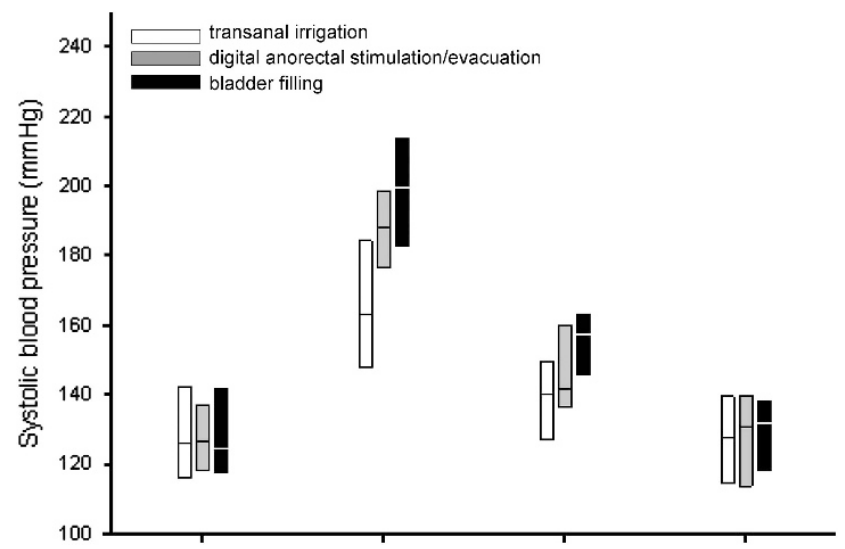

Figure 1 Systolic blood pressure (median and range) during bladder filling (black), transanal colorectal irrigation (white) and digital anorectal stimulation/evacuation (gray) in subjects with high $\mathrm{SCl}$. Results are given for baseline, maximum stimulation (Max.stim.), $3 \mathrm{~min}$ after maximum stimulation ( $+3 \mathrm{~min})$ and $12 \mathrm{~min}$ after maximum stimulation $(+12 \mathrm{~min})$. The boxes are displayed slightly offset for better reader friendliness.
Transanal colorectal irrigation is increasingly used for the management of neurogenic bowel dysfunction and the clinical effect has been documented in a European, randomized, multicenter trial. ${ }^{8}$ During transanal irrigation, $500-1000 \mathrm{ml}$ are administered to the rectum and distal colon. Before the present study, it was unknown whether this could prove a strong stimulus for $\mathrm{AD}$ and if patients with a history of $\mathrm{AD}$ should be restricted from the use of irrigation. Our data indicate that irrigation causes less severe $\mathrm{AD}$ than bladder filling and standard digital anorectal stimulation. This was somewhat surprising, but the data are supported by a previously reported trend towards less symptoms of $\mathrm{AD}$ during irrigation as compared with standard bowel management. ${ }^{8}$

Episodes of $\mathrm{AD}$ are frequently associated with various signs and symptoms that could be life threatening. ${ }^{2}$ Although numerous mechanisms for the development of $\mathrm{AD}$ have been proposed and examined, the pathophysiology is still not completely understood. ${ }^{11}$ The clinical hallmark of AD is an abrupt increase of sBP and usually the definition is a rise of at least 20 or $30 \mathrm{~mm} \mathrm{Hg}$ above baseline pressure. ${ }^{17}$ The sympathetic discharge during $\mathrm{AD}$ is triggered by noxious or nonnoxious stimuli below the level of lesion. ${ }^{18}$ Recent data from animal studies suggest that TRPV1 (transient receptor potential vanilloid 1)-mediated stimuli from the colorectum are involved in conveying the stimuli that trigger episodes of $\mathrm{AD} .{ }^{19}$ Axons of the sensory neurons from the thoracolumbar and lumbosacral dorsal root ganglia expressing TRPA1 (transient receptor potential ankyrin 1) are present in the pelvic nerves that reach the distal colon. ${ }^{20}$ It is believed that TRPV1 neurons are involved in the regulation of colonic motor function, but usually not in nociception. ${ }^{21}$ TRPA1 is necessary for normal mechanosensory function and the signaling of noxious mechanical stimuli. ${ }^{21}$ It remains to be shown whether stimulation of other colorectal mechanoreceptors causes AD.

In contrast to the colon and the rectum, the anal canal is densely innervated by afferent nerve fibers. This may explain our observation that digital anorectal stimulation was a more powerful stimulus for $\mathrm{AD}$ than transanal colorectal irrigation. As two-thirds of subjects with SCI use digital anorectal stimulation or evacuation, and $37 \%$ of patients have mild symptoms of $\mathrm{AD}$ during bowel evacuation, ${ }^{5}$ a clinical

Table 4 Autonomic response to bowel and bladder procedures

\begin{tabular}{|c|c|c|c|c|}
\hline & Baseline & Maximum stimulation & At 3 min after maximum stimulation & At 12 min after maximum stimulation \\
\hline \multicolumn{5}{|l|}{ Transanal irrigation } \\
\hline $\mathrm{sBP}(\mathrm{mm} \mathrm{Hg})$ & $126(91-146)$ & $164(130-188)^{*}$ & $140(103-152)^{*}$ & $128(104-145)$ \\
\hline $\mathrm{dBP}(\mathrm{mm} \mathrm{Hg})$ & $73(61-87)$ & $91(76-101)^{*}$ & $80(68-125)^{*}$ & $77(50-96)$ \\
\hline HR (b.p.m.) & $71(56-94)$ & $66(53-91)$ & $71(56-101)$ & $77(50-113)$ \\
\hline Norepinephrine (nmoll-1) & $2.66(0.49-3.52)$ & - & $2.965(0.47-4.17)^{*}$ & $3.09(0.5-3.55)$ \\
\hline Epinephrine $\left(\mathrm{nmol} \mathrm{I}^{-1}\right)$ & $0.055(0.03-0.22)$ & - & $0.05(0.03-0.42)$ & $0.08(0.009-0.51)$ \\
\hline \multicolumn{5}{|l|}{ Digital anorectal stimulation } \\
\hline $\mathrm{sBP}(\mathrm{mm} \mathrm{Hg})$ & $127(86-154)$ & $188(140-206)^{*}$ & $142(102-167)^{*}$ & $131(83-147)$ \\
\hline $\mathrm{dBP}(\mathrm{mm} \mathrm{Hg})$ & $76(60-96)$ & $110(87-134)^{*}$ & $90(66-102)^{*}$ & $75(47-89)$ \\
\hline HR (b.p.m.) & $81(60-98)$ & $72(32-98)$ & $82(52-98)$ & $83(53-95)$ \\
\hline Norepinephrine (nmoll-1) & $2.26(0.049-3.06)$ & - & $3.04(0.4-3.86)^{*}$ & $2.24(0.33-3.42)$ \\
\hline Epinephrine $\left(\mathrm{nmol} \mathrm{I}^{-1}\right)$ & $0.09(0.02-0.14)$ & - & $0.08(0.03-0.24)$ & $0.055(0.009-0.14)$ \\
\hline \multicolumn{5}{|l|}{ Bladder filling } \\
\hline $\mathrm{sBP}(\mathrm{mm} \mathrm{Hg})$ & 125 (106-149) & $200(179-220)^{*}$ & $158(142-172)^{*}$ & $132(105-153)$ \\
\hline $\mathrm{dBP}(\mathrm{mm} \mathrm{Hg})$ & $77(54-90)$ & $109(80-127)^{*}$ & $87(67-106)^{*}$ & $79(64-86)$ \\
\hline HR (b.p.m.) & $74(46-105)$ & $60(41-99)$ & $66(42-103)$ & $73(49-113)$ \\
\hline Norepinephrine (nmoll-1) & $2.4(0.39-4.28)$ & - & $2.91(0.87-5.95)^{*}$ & $2.81(0.37-5.95)$ \\
\hline Epinephrine $\left(\mathrm{nmol} \mathrm{I}^{-1}\right)$ & $0.05(0.009-0.17)$ & - & $0.06(0.009-1.02)$ & $0.07(0.009-0.26)$ \\
\hline
\end{tabular}

Abbreviations: b.p.m., beats per min; $\mathrm{dPB}$, diastolic blood pressure; HR, heart rate; $\mathrm{sBP}$, systolic blood pressure.

Autonomic response to transanal colorectal irrigation, digital anorectal stimulation and bladder filling in the eight subjects with high spinal cord injury is shown. All these eight subjects with high spinal cord injury had autonomic dysreflexia during maximum stimulation, but sBP had returned to baseline 12 min after maximum stimulation.

*Statistically significant increase compared with baseline values $(P<0.05)$. 
implication of our study is that TAIcould be considered as an alternative in patients with severe $\mathrm{AD}$ during digital bowel evacuation.

Numerous previous studies documented that resting catecholamine levels, particularly norepinephrine, are low following cervical SCI. This is due to sympathetic hypoactivity. ${ }^{22}$ However, during the episodes of $\mathrm{AD}$ there is activation of the spinal sympathetic circuits that result in prompt elevation in levels of plasma norepinephrine but not epinephrine. Similar to previous observations, AD in our subjects was also associated with significant increase in serum norepinephrine levels. Although there were significant differences in the severity of $\mathrm{AD}$ episodes between the various procedures tested, this was not reflected by the levels of the norepinephrine.

Episodes of $\mathrm{AD}$ are usually short lasting once the stimulus is removed. Our data support the clinical approach of immediately removing the stimulus for $\mathrm{AD} .^{23}$ Thus, $\mathrm{sBP}$ and elevated blood levels of norepinephrine quickly fell as soon as stimulation was stopped and returned to baseline within $12 \mathrm{~min}$. The risks associated with $\mathrm{AD}$ are difficult to estimate. Intracerebral hemorrhage and deaths have been reported, but $\mathrm{AD}$ may be silent and some cases of sudden unexplained death in subjects with SCI may be caused by AD. Usually, the severity of $\mathrm{AD}$ is described as the maximum change in sBP but 'time at risk' with sBP above a certain level may be equally important for risk analysis. Numerous treatments of $\mathrm{AD}$ have been attempted. ${ }^{24}$ Of special relevance to the present study, topical administration of lidocaine may be attempted in patients with repeatedly severe $\mathrm{AD}$ in response to digital anorectal stimulation.

For the present study we applied bladder and bowel stimuli as close to our clinical practice as possible. Thus, digital anorectal stimulation/ evacuation was performed as proposed by Coggrave and Norton, ${ }^{14}$ TAI was done with a standardized volume and inflow and bladder filling was done as during cystometry. Individual patients may have daily routines different from those used in the present study and, in general, bowel emptying procedures performed at home were more time consuming than allowed for in the present study. Therefore, we may have underestimated the severity of $\mathrm{AD}$ during digital anorectal stimulation/evacuation. In the present study we used $500 \mathrm{ml}$ for irrigation. In daily practice, most patients use between 500 and $1000 \mathrm{ml}$ and we may have underestimated the autonomic response to TAI in patients using higher volumes than $500 \mathrm{ml}$. Baclofen passes the blood-brain barrier very slowly, and with the use of intrathecal baclofen the concentration in plasma does rarely exceed $5 \mathrm{ng} \mathrm{ml}^{-1}$. This was considered of little consequence for the present study.

Our study population was mainly selected among patients with a history $\mathrm{AD}$, and the results are probably not applicable to the whole population of patients with high SCI. As our aim was to compare responses with the predefined stimuli, we do not find that the validity of conclusions is hampered by selection bias.

In conclusion, we found that TAI is a less severe stimulus for $\mathrm{AD}$ than bladder filling or digital anorectal stimulation. Consequently, we do not find that previous symptoms of $\mathrm{AD}$ should restrict patients from treatment with irrigation if it is tolerated during initial administrations. Knowledge about $\mathrm{AD}$ and treatment thereof is important to doctors and staff introducing patients to treatment of neurogenic bowel disorders and further studies of mechanisms and neuronal pathways responsible for the development of $\mathrm{AD}$ in response to stimuli of pelvic organs are needed.

\section{DATA ARCHIVING}

There were no data to deposit.

\section{CONFLICT OF INTEREST}

The authors declare no conflict of interest.

\section{ACKNOWLEDGEMENTS}

We thank nurses Gitte Sørensen, Lotte Jacobsen, Margit Majgaard, Birgitte Kvitzau and Birgitte Gustafson. PMF performed the research; PMF, PC and KK analyzed the data and wrote the paper; EF provided blood analysis; PMF, PC, $\mathrm{AK}, \mathrm{SL}$ and $\mathrm{KK}$ designed the research study and wrote the paper.

1 Teasell RW, Arnold JM, Krassioukov A, Delaney GA. Cardiovascular consequences of loss of supraspinal control of the sympathetic nervous system after spinal cord injury. Arch Phys Med Rehabil 2000; 81: 506-516.

2 Consortium for Spinal Cord Medicine. Acute management of autonomic dysreflexia: individuals with spinal cord injury presenting to health-care facilities. J Spinal Cord Med 2002; 25(Suppl 1), S67-S88.

3 Krassioukov A, Claydon VE. The clinical problems in cardiovascular control following spinal cord injury: an overview. Prog Brain Res 2006; 152: 223-229.

4 Ekland MB, Krassioukov AV, McBride KE, Elliott SL. Incidence of autonomic dysreflexia and silent autonomic dysreflexia in men with spinal cord injury undergoing sperm retrieval: implications for clinical practice. J Spinal Cord Med 2008; 31: 33-39.

5 Krogh K, Nielsen J, Djurhuus JC, Mosdal C, Sabroe S, Laurberg S. Colorectal function in patients with spinal cord lesions. Dis Colon Rectum 1997; 40: 1233-1239.

6 Consortium for Spinal Cord Medicine. Bladder management for adults with spinal cord injury. A clinical practice guideline for health-care providers. J Spinal Cord Med 2006 29: 527-573.

7 Faaborg PM, Christensen P, Finnerup N, Laurberg S, Krogh K. The pattern of colorectal dysfunction changes with time since spinal cord injury. Spinal Cord 2008; 46 234-238.

8 Christensen P, Bazzocchi G, Coggrave M, Abel R, Hultling C, Krogh K et al. A randomized, controlled trial of transanal irrigation versus conservative bowel management in spinal cord-injured patients. Gastroenterology 2006; 131: 738-747

9 Christensen P, Olsen N, Krogh K, Bacher T, Laurberg S. Scintigraphic assessment of retrograde colonic washout in fecal incontinence and constipation. Dis Colon Rectum 2003; 46: 68-76

10 Faaborg PM, Christensen P, Kvitsau B, Buntzen S, Laurberg S, Krogh K. Long-term outcome and safety of transanal colonic irrigation for neurogenic bowel dysfunction. Spinal Cord 2009; 47: 545-549.

11 Krassioukov A. Autonomic function following cervical spinal cord injury. Respir Physiol Neurobiol 2009; 169: 157-164.

12 Krogh K, Perkash I, Stiens SA, Biering-Sorensen F. International bowel function basic spinal cord injury data set. Spinal Cord 2009; 47: 230-234.

13 Alexander MS, Biering-Sorensen F, Bodner D, Brackett NL, Cardenas D, Charlifue S et al. International standards to document remaining autonomic function after spinal cord injury. Spinal Cord 2009; 47: 36-43.

14 Coggrave MJ, Norton C. The need for manual evacuation and oral laxatives in the management of neurogenic bowel dysfunction after spinal cord injury: a randomized controlled trial of a stepwise protocol. Spinal Cord 2009; 48: 504-510.

15 Huang YH, Bih LI, Chen GD, Lin CC, Chen SL, Chen WW. Autonomic dysreflexia during urodynamic examinations in patients with suprasacral spinal cord injury. Arch Phys Med Rehabil 2011. 92: 1450-1454.

16 Furusawa K, Tokuhiro A, Sugiyama H, Ikeda A, Tajima F, Genda E et al. Incidence of symptomatic autonomic dysreflexia varies according to the bowel and bladder management techniques in patients with spinal cord injury. Spinal Cord 2011; 49: 49-54.

17 Spinal Cord Injury Rehabilitation Evidence, version 4.0, https://www.scireproject.com 2010.

18 Furlan JC, Urbach DR, Fehlings MG. Optimal treatment for severe neurogenic bowel dysfunction after chronic spinal cord injury: a decision analysis. Br J Surg 2007; 94: 1139-1150.

19 Ramer LM, van Stolk AP, Inskip JA, Ramer MS, Krassioukov AV. Plasticity of TRPV1expressing sensory neurons mediating autonomic dysreflexia following spinal cord injury. Front Physiol 2012; 3: 257.

20 Nilius B, Appendino G, Owsianik G. The transient receptor potential channel TRPA1: from gene to pathophysiology. Pflugers Arch 2012; 464: 425-458.

21 Suckow SK, Anderson EM, Caudle RM. Lesioning of TRPV1 expressing primary afferent neurons prevents PAR-2 induced motility, but not mechanical hypersensitivity in the rat colon. Neurogastroenterol Motil 2012; 24: e125-e135.

22 Claydon VE, Krassioukov AV. Orthostatic hypotension and autonomic pathways after spinal cord injury. J Neurotrauma 2006; 23: 1713-1725.

23 Krassioukov A, Eng JJ, Warburton DE, Teasell R. A systematic review of the management of orthostatic hypotension after spinal cord injury. Arch Phys Med Rehabil 2009, 90: 876-885.

24 Krassioukov A, Warburton DE, Teasell R, Eng JJ. A systematic review of the management of autonomic dysreflexia after spinal cord injury. Arch Phys Med Rehabil 2009, 90: 682-695. 